\title{
Wissenschaftliche Expertise und die (Re)Produktion gesellschaftlicher Verhältnisse - Eine argumentationstheoretische Analyse von Diskursen der Ökologie- und Umweltforschung im westlichen China
}

\author{
Ferdinand Stenglein \\ Institute of Sociology, University of Münster, 48151 Münster, Germany \\ Correspondence to: Ferdinand Stenglein (f_sten08@uni-muenster.de)
}

Received: 2 September 2016 - Revised: 17 January 2017 - Accepted: 25 February 2017 - Published: 3 May 2017

\begin{abstract}
Kurzfassung. On the basis of the analysis of scientific publications on environmental issues and issues of ecology in Western China, I show in this contribution how scientific expertise can be analyzed as an order of arguments and its social embeddedness be discussed. Using the example of environmental science in Western China, an order of knowledge is reconstructed by coding techniques and the axiomatic structure of this order is micro-analytically disclosed using argumentation analysis. Subsequently, this order is discussed in relation to the social context within which it evolved. Normative representations of subjectivities, practices and spatial arrangements are produced with references to an ideal of "harmony" in the analyzed publications. Against the background of the contested social relations in Western China, it becomes clear that these representations are involved with a cultural recomposition and contribute to stabilizing state control and national integrity in the region and reproducing unequal social relations. The analysis shows how allegedly neutral academic knowledge implicitly (re)produces social orders and thus stresses that there can be no scientific expertise that does not take a normative position. Lastly, this raises questions on why, how and with which effects we engage in research and produce scientific knowledge.
\end{abstract}

\section{Die Ökologie- und Umweltforschung im westlichen China}

Eines der zentralen Forschungsfelder im westlichen China ist die Ökologie- und Umweltforschung. Mehrere nationale und internationale Forschungsprojekte, wie beispielsweise das Forschungskonsortium SuMaRiO (2016), sind dort mit großvolumigen Forschungsvorhaben vertreten. Diese Forschungsvorhaben können in die, im Jahre 1999 von der chinesischen Zentralregierung ausgerufene, Great Western Development Strategy (GWDS) eingeordnet werden. Diese zielt darauf ab einen wirtschaftlichen „catch-up“ Prozess in den westlichen Regionen Chinas zu befördern. Denn diese sogenannte westliche „Peripherie“ ist wirtschaftlich von wesentlich geringerer Bedeutung als die küstennahen und exportorientierten östlichen Regionen des Nationalstaats China (Veeck et al., 2007). Innerhalb der GWDS spielt dabei die ökologische Ausrichtung wirtschaftlicher Aktivitäten eine entscheidende Rolle. So werden im Rahmen der GWDS großformatige, staatliche Umweltsanierungsprogramme, wie das National Forest Protection Programme (NFPP) oder das Slope Land Conversion Programme (SLCP) umgesetzt (Yeh, 2005:11). Die ökologische Umstrukturierung der chinesischen Wirtschaft und Gesellschaft wird dabei zunehmend zu einer zentralen Staatsraison und einem der wichtigsten Forschungsfelder der chinesischen Forschungslandschaft (Mol, 2006) (vgl. dazu beispielsweise auch die Rede des chinesischen Umweltministers Zhou, 2012).

Die Wichtigkeit wissenschaftlicher Arbeiten zu MenschUmwelt-Beziehungen nimmt dabei insbesondere im Westen Chinas einen zentralen Status ein. Denn gerade dieser Region werden die größten ökologischen Probleme zugeschrieben und als Gefahr für die wirtschaftliche Entwicklung des Westens und der gesamten Nation gesehen. So werden die 
Ursachen von Umweltkatastrophen im Osten Chinas, wie beispielsweise die große Flut des Yangtse Flusses im Jahre 1998, dem Management der Landnutzung in den westlichen Regionen Chinas zugeschrieben (Yeh, 2005). Die Forschungsarbeiten zu Mensch-Umwelt-Beziehungen drehen sich im Südwesten, den Hochgebirgsregionen der Provinz Yunnan und der Autonomen Region Tibet, dabei um Themen wie Hangerosion und Weidewirtschaft oder die Nutzung von Ökosystemdienstleistungen. In den ariden Gebieten des Nordwestens, der Autonomen Region Xinjiang, liegt das Hauptinteresse der Forschung in der Ressource „Wasser", deren Nutzung und Verteilung, sowie Desertifikationsprozessen.

Gleichzeitig finden diese Forschungsarbeiten in einem Kontext statt, der von großen sozialen Spannungen zwischen han-chinesischer, tibetischer und uighurischer Bevölkerung gekennzeichnet ist. Wiederholt kam es in den letzten Jahren in den westlichen Regionen zu gewaltsamen und tödlichen Zusammenstößen dieser Bürger*innen mit hanchinesischer Bevölkerung und den Polizei- und Militärapparaten der Volksrepublik China (VRC) (Wong, 2009). Gleichzeitig werden massive Umsiedlungs-, Infrastruktur- und Investitionsprogramme der Regierung implementiert und ändern die soziale und materielle Zusammensetzung und Struktur der westlichen Regionen. Die Umwelt- und Ökologieforschung ist dort folglich in einem höchst umkämpften gesellschaftlichen Kontext verortet und eingebettet in die zentralistische Forschungslandschaft Chinas, in der die staatliche Forschungsakademie (Chinese Academy of Sciences, CAS) eine dominante Stellung bei der Durchführung und Finanzierung von Forschung einnimmt.

Vielfach wurde gezeigt, dass Umweltforschung und Umweltwissen nicht unbeeindruckt von ihrem gesellschaftlichen Kontext geblieben sind, sondern im Gegenteil eine aktive, gouvernmentale Rolle einnehmen können (Blaikie und Muldavin, 2004; Forsyth, 2003; Yeh, 2005). Forsyth und Walker (2008:229, eigene Übersetzung) konstatieren in ihrer Analyse von umweltbezogenen Narrativen in Thailand, „Umweltwissen existiert nicht in politisch neutraler und universell anwendbarer Form, sondern reflektiert verschiedene soziale und politische Einflüsse und Werte“. Im Licht dieser Studien stellt sich folglich für das Umweltwissen der Umwelt- und Ökologieforschung in China nicht die Frage ob, sondern auf welche Weise ihre Wissensproduktion in die gesellschaftlichen Verhältnisse involviert ist und wie dies untersucht werden kann. In ihren Studien zur Umweltpolitik in China hat Emily Yeh $(2005,2009)$ bereits angedeutet, dass das "Greening“ von Chinas Westen und der chinesischen Gesellschaft im Allgemeinen zu weitreichenden Verschiebungen der sozialen Verhältnisse beiträgt. Dies soll hier im Blickfeld der Wissenschaftsforschung weiter konkretisiert werden. Jasanoff (2005) identifiziert dabei drei Felder, auf die sich eine Analyse der Rolle wissenschaftlicher Expertise richten kann: Dies sind erstens Akteure (wissenschaftliche Expert*innen), zweitens Vermittlungsinstitutionen von
Expertise (Kommissionen, Beiräte, etc.) und drittens akademische Wissensordnungen (Publikationen, Forschungsdesigns, etc.). Diese Dreiteilung ist hilfreich dafür, einen analytischen Zugangspunkt zur Thematisierung der sozialen Einbettung von Forschungsarbeiten zu wählen. Diese analytische Vereinfachung sollte aber nicht dazu verleiten Personen, Institutionen und Wissensproduktion als voneinander losgelöst zu betrachten.

Im vorliegenden Beitrag wurde aus pragmatischen Grün$\operatorname{den}^{1}$ ein primärer Zugang über die Analyse von Wissensordnungen gewählt und ca. 100 englischsprachige, wissenschaftliche Publikationen des entsprechenden Forschungsfelds aus den Jahren 1999-2013 asynchron untersucht. Aus dem Korpus an Publikationen wurden dabei hegemoniale Diskurse auf Basis eines argumentationstheoretischen Vorgehens rekonstruiert (z.B. Glasze et al., 2009) und mikroanalytisch entlang der diskursanalytisch erweiterten Argumentanalysen Stephen Toulmins (z.B. Felgenhauer, 2015) untersucht. In einem weiteren Schritt wurden die Ergebnisse dieser Analyse mit einem Korpus nicht-wissenschaftlicher Publikationen interpretativ diskutiert (Diaz-Bone, 2006; Landwehr, 2009). Auf Grundlage dieses Vorgehens konnte herausgearbeitet werden, wie die hegemoniale Wissensordnung der untersuchten wissenschaftlichen Publikationen durch räumliche Repräsentationen und die Repräsentation von „guten“ und „schlechten“ Subjekten und Praktiken die territoriale Kontrolle des chinesischen Nationalstaats stabilisiert und legitimiert und damit den han-chinesischen Eliten des Landes zu Gute kommt. So konnte durch die Analyse auch gezeigt werden, dass und wie die analysierten Publikationen ,sozial konditioniert" (Fuller nach Keith und Rehg, 2008:225, eigene Übersetzung) sind. Letztlich zeigen sich in ihnen die Möglichkeitsräume der Verbreitung, Durchführung, Verwendung, Akzeptanz, Relevanz und Aneignung, kurz die sozialen Herrschafts- und Machtverhältnisse in denen und als Ausdruck derer sich die Ökologie- und Umweltforschung artikuliert.

\section{Wissenschaft als Diskurse der Argumentation}

Argumentation ist eine wesentliche Tätigkeit wissenschaftlichen Arbeitens. Mit ihr werden Behauptungen auf Grundlage von Evidenzen aufgestellt. Über Argumentation wird es möglich einen Schluss von einem bestimmten Faktum zu ziehen. So können durch Argumentationen Behauptungen über die Beschaffenheit der Dinge (z.B. Theorien) gegenüber Zweifeln an diesen Aussagen verteidigt und gleichzeitig Kontroversen über Behauptungen beigelegt werden. Argumentation ist damit eine zentrale wissenschaftliche Technik, um die Plausibilität wissenschaftlicher Aussagen zu begründen und wissenschaftliche Objektbereiche zu formieren (Evagorou und Osborne, 2010:154). Dabei werden nicht nur

\footnotetext{
${ }^{1}$ Ethnographische Feldstudien konnten nicht erfolgreich durchgeführt werden.
} 
in Publikationen Argumente ausgearbeitet, um Thesen zu plausibilisieren, sondern auch die Praktiken der Herstellung empirischer Evidenzen müssen über Argumentationen legitimiert werden. Über Argumentationen wird so wissenschaftliches Wissen aufgebaut, verschoben und in Frage gestellt. Ein möglicher Zugang zur Analyse von ,Wissenschaft“ bietet sich folglich darüber an, sie als durch Argumente und Argumentationsketten formierte Wissensordnungen zu untersuchen und auf diese Weise wissenschaftliche Diskurse, deren Verhältnisse zueinander und deren implizite Setzungen zu identifizieren.

Ideengeschichtlich wurden Argumente zunächst als Phänomen formaler Logik und Phänomen der Rhetorik verstanden und untersucht. Eine solche Analyse von Argumenten zielt beispielsweise darauf ab, auf welche Weise der konkrete semantische oder situative Kontext eines gesprochenen oder geschriebenen Arguments dieses logisch werden lässt oder welche rhetorischen Mittel Argumentationen überzeugend machen (vgl. Eemeren et al., 2014; Wintzer, 2014:113). Es wird also die Binnenlogik von Argumenten untersucht (z.B. Amossy, 2009).

Die von mir verfolgte Fragestellung nach der gesellschaftlichen Herstellung und Machtförmigkeit akademischer Wissensbestände macht einen anderen primären Zugang zu Argumentation notwendig. Um die gesellschaftliche Eingebettetheit von Argumentationen aufzeigen zu können sind Argumente hier nicht als konkrete argumentative Überzeugungsstrategien durch ihre formale Logik, textuelle oder semantische Stellung interessant, sondern in der Art und Weise, wie durch sie Wissensordnungen hergestellt werden und sie bedeutungskonstitutiv wirken. Eine solche konstruktivistische Perspektive auf Argumente zielt darauf, aus einem Korpus an (Text)Dokumenten dessen argumentative Strukturiertheit zu rekonstruieren und deren kontingente Grundlagen dekonstruierend sichtbar zu machen. Dem zu Grunde liegt eine Ontologie, die davon ausgeht, dass sich unser Zugang zur Welt über Bedeutung ergibt. „Dinge“ werden „Dinge“ dadurch, wie wir sie benennen und wie sie gegenüber anderen Dingen abgegrenzt und in eine spezifische Qualität zu diesen gesetzt werden. Letztlich folgt aus diesem Verständnis, dass keine sichere Basis dafür gegeben ist, wie uns Wirklichkeit erscheint und damit wie Wirklichkeit ist (bzw. genannt wird zu sein). In diesem Sinne ist diese Ontologie ontologisch leer (Laclau und Mouffe, 2001:186). Konstruktivistisch ist dieses Verständnis, da es konsequenterweise keine objektive Grundlage außerhalb der Repräsentation von Wirklichkeit geben kann. Wirklichkeit als ein Muster an Repräsentationen, muss sich somit aus den Beziehungen des Benennens selbst ergeben (Marttila, 2015b:36).

Auf Grundlage einer solchen Ontologie, bezeichnet „Diskurs" die Geflechte an Beziehungen, die Wirklichkeit konstituieren. Diskurse definieren damit immer wieder die Ausgangsbedingungen dafür, wie uns Dinge erscheinen und streitbar werden (Foucault, 1981:74). Argumentation ist aus dieser Warte eine spezifische Technologie und Perspekti- ve neben anderen (z.B. Aussagen, Narrationen) durch die sich Diskurse formieren, Ordnung hergestellt wird und diese Konstruktionen untersucht werden können.

Auch für Argumente kann es folglich in dieser Perspektive keine außerargumentative, objektive (bzw. rationale) oder transzendente Basis geben, auf die sie sich gründen können. Die Stichhaltigkeit eines Arguments ergibt sich ausschließlich aus den Beziehungen zu anderen Argumenten. Analytisch von primärem Interesse ist damit der weiter gespannte, interpretative Kontext der Beziehungen von Argumentationen und Argumentationsketten, der die Stichhaltigkeit eines Arguments gewährleistet. Argumente werden somit nicht als logische Operationen aufgefasst, sondern als informelle Inferenzen (Felgenhauer, 2009:276; Wintzer, 2014:214). Sie können nur deshalb Strategien der Überzeugung sein, da sie sich auf einem bestimmtem impliziten Hintergrundwissen und Begründungszusammenhängen gründen (Tans, 2006). Im Unterschied zur Betrachtung der Binnenlogik von Argumenten, beziehen Argumente auf Grundlage einer konstruktivistischen Ontologie ihre Logik aus einem letztlich kontingenten, diskursiven Möglichkeitskontext. Analytisch wird aus dieser Perspektive also angestrebt die Konstitutionsbedingungen, die Argumente und Argumentationsketten überzeugend und konsistent machen, herauszuarbeiten (vgl. Felgenhauer, 2007; Glasze und Mattissek, 2014; Wintzer, 2014). Eine solche Analyse von Argumenten in der Wissenschaft zielt folglich darauf ab, Wissenschaft ,als diskursive Formation[en] in Frage zu stellen; es heißt, nicht gegen die formalen Widersprüche ihrer Propositionen anzugehen, sondern gegen das Formationssystem ihrer Gegenstände, ihrer Äußerungstypen, ihrer Begriffe, ihrer theoretischen Wahlmöglichkeiten“(Foucault, 1981:265). ${ }^{2}$

\section{Re- und Dekonstruktive Argumentanalyse}

Aus dem Verständnis von Wissenschaft als Diskurse der Argumentation ergeben sich zwei analytische Grundbewegungen: Erstens wird in einer Rekonstruktionsbewegung danach gefragt, wie sich Argumente aufeinander beziehen und welches Strukturgeflecht sich dabei rekonstruieren lässt. Ziel dieser Analyse ist es, über die Beziehungsgeflechte von Argumentationen und Argumentationsketten ein Regime an Argumentationen herauszuarbeiten, das eine herausragende und dominante Stellung bei der Produktion von Wirklichkeit einnimmt. Zweitens wird in einer dekonstruierenden Bewegung gefragt, welche objektivierten Bedingungen die Argumentationsketten dieses Regimes als logisch erscheinen lassen. Welches sind seine impliziten, ersten Prämissen und Axiome? Die Analyse entfaltet sich entlang dieser Fragen somit als eine Rekonstruktion von Strukturiertheit und eine De-

\footnotetext{
${ }^{2}$ Die Übersetzung von „attaquer“ als „,angehen gegen“ ist unglücklich gewählt. Aus meiner Sicht ist ,attaquer“ im Sinne einer kritischen Befragung gemeint.
} 
konstruktion der vermeintlichen Notwendigkeit dieser Struktur.

Wichtigste methodologische Voraussetzung der Analyse ist dabei die Durchführung eines epistemologischen Bruchs. Durch den epistemologischen Bruch wird der Abstraktionssprung vom empirischen Material (hier Texte des Korpus) auf diese konstituierenden Diskurse repräsentiert. Diese gedankliche und analytische Trennung ist notwendig, da nicht direkt vom empirischen Material ,erster Ordnung“ auf die dahinter liegenden diskursiven Regeln und diskursive Strukturiertheit geschlossen werden kann, die diese Praktiken zu sinnhaften Praktiken machen (Ebene ,zweiter Ordnung“). Dies zeigt nochmals deutlich, wodurch sich eine konstruktivistische Herangehensweise an Argumentationen auszeichnet. Macht-Wissen-Komplexe können nicht durch hermeneutische Interpretation oder intersubjektive Sinnanalyse von Gesagtem und Geschriebenem direkt analysiert werden. Vielmehr wird erst durch einen Abstraktionsbruch von sinnhaften Praktiken (hier Argumentationen in Texten) auf deren implizite Hintergrundskripte, deren Formationssysteme, geschlossen (Diaz-Bone, 2008; Glasze et al., 2009:294; Marttila, 2015b:106 passim).

Aus der Vielzahl an Möglichkeiten der methodischen Gestaltung von Diskursanalysen (Glasze und Mattissek, 2009, 2014), werden hier kodierende Verfahren (Glasze et al., 2009) mit den an Stephen Toulmin anschließenden, dekonstruktiven Argumentanalysen von Tilo Felgenhauer (2007, 2009, 2015) kombiniert.

\subsection{Begründungen dekonstruktiv herausarbeiten}

Vor allem Stephen Toulmin hat in den 1950er Jahren einen Neuanfang der Argumentationstheorie eröffnet, welcher eine Annäherung an ein konstruktivistisches Verständnis von Argumentation erlaubt (Wintzer, 2014:116). Toulmin wendet sich gegen die Analyse formaler Logik von Argumentationen und entwickelt einen Ansatz, der auf die letzten und implizit konventionell begründeten Elemente von Argumenten abstellt (Eemeren et al., 2014:203 passim; Wintzer, 2014:117).

Nach Toulmin (2003) können Argumente analytisch durch „Daten“, „Behauptung“, „Schlussregel“" und „Backing“ gefasst werden (Abb. 1). „Daten“ bilden die Grundlage für eine Behauptung, eine Schlussfolgerung, die aus den Daten gezogen wird. Diese binäre Beziehung konstituiert das Argument. Um allerdings eine Schlussfolgerung ziehen, also eine Behauptung aufstellen zu können, müssen bestimmte Regeln gelten, die diese Deduktion gültig machen. Diese „Schlussregeln“ legitimieren den vermuteten Schritt von „Daten“ zu „Behauptung“. Allerdings wird so noch nicht deutlich, warum eine „Schlussregel“ Autorität zu dieser Legitimierung besitzen sollte. Schlussregeln sind nach Toulmin folglich selbst in Hintergrundannahmen, „Backings“, fundiert (Toulmin, 2003:87). ,,Schlussregel“ und „Backing“ sind damit keine Interpretationen, sondern implizite Elemente des Arguments und reflektieren kollektiv geteilte, konventionel-
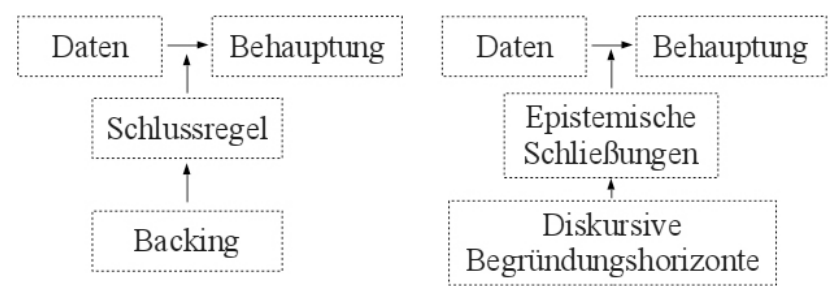

Abb. 1. Links: Toulmin Modell nach Toulmin (2003:87); rechts: diskurstheoretisch erweitertes Modell.

le Wissensbestände (Felgenhauer, 2009:273). Sie wirken als Konsistenzbedingungen des Arguments. „Backings“ sind damit feldabhängig, bzw. es wird deutlich, dass ,Schlussregel“" und „Backing“ selbst Produkte von Argumentationen sind. Einerseits sind sie so die kontestierbaren und veränderlichen, letztlich kontingenten Fundierungen von Argumenten und andererseits sind sie selbst temporäre, aber weitgehend normalisierte und sedimentierte Resultate von Argumentationen. So kann für die Analyse von wissenschaftlichen Argumenten „Backing“ als Verankerung von Argumenten in impliziten und letztlich kontingenten Axiomen und die „Schlussregel“ als epistemisch informierte Schließung dieser diskursiven Verankerung konzeptionalisiert werden (Abb. 1) (vgl. dazu auch Felgenhauer, 2009:266 passim, 2015:50; Wintzer, 2014:118). Aus methodologischer Sicht wird damit eine Abstraktion von der direkten Argumentebene (Bereich erster Ordnung) auf die implizite Ebene der Begründung dieser Argumentation (Bereich zweiter Ordnung) vorgenommen. Was als Wissenschaft gilt und welche Argumente folglich als legitime wissenschaftliche Argumente gelten, ist zum einen eine Frage der epistemischen SchlieBung durch Methodik und Methodologie und die Produktion konkreter legitimer Daten und legitimer Interpretationen. Zum anderen ist es eine grundlegende Frage danach, welche Fragen überhaupt begründet gestellt werden (können) und in welchem Rahmen diese Fragen gestellt werden (können).

Mit dem hier vorgestellten Analyseschema, kann mikroanalytisch herausgearbeitet werden, unter welchen Bedingungen einzelne Argumente des analysierten Textkorpus als Argumente gültig werden und in welchem Argumentraum sich diese bewegen. Dieses Vorgehen ermöglicht für ausgewählte, exemplarische Argumente des Korpus herauszuarbeiten, welche Formationssysteme und ersten Begründungen das jeweilige Argument gültig machen und worauf sich Argumente in den analysierten Textdokumenten beziehen (z.B. die naturalisierte Vorstellung eines homo oeconomicus). Es ist davon auszugehen, dass sich im Korpus mehrere dieser diskursiven Begründungshorizonte identifizieren lassen, die die verschiedenen mit einander in Beziehung stehenden Argumente des Korpus jeweils sinnhaft werden lassen. So ermöglicht diese Argumentanalyse über die Argumente des Korpus hinaus dessen axiomatische Struktur und dessen 
Bezüge zu unterschiedlichen Diskursfeldern dekonstruierend offen zu legen.

Um das Zusammenwirken, und damit die Strukturiertheit verschiedener Argumente und ihrer Begründungshorizonte im Korpus zu einander deutlich $\mathrm{zu}$ machen, werden parallel zu dieser Mikroanalyse Argumentabschnitte kodiert und daraus eine Ordnungsstruktur rekonstruiert.

\subsection{Argumentationsordnungen kodieren und rekonstruieren}

Prinzipiell können Rekonstruktionen mit unterschiedlichsten Methoden umgesetzt werden (vgl. Glasze und Mattissek, 2009, Kapitel C). Die Wahl der Methode hängt dabei entscheidend von den Forschungsfragen, der analytischen Reichweite und der Materialbeschaffenheit ab. Hier wurde ein qualitativ-kodierendes Verfahren gewählt, das sich in seinem Vorgehen an der explorativen Methodik der Grounded Theory (GTM) orientiert. Diese muss den methodologischen Voraussetzungen einer Diskursanalyse angepasst werden. Insbesondere wird dabei der Stellenwert des Kodierens verändert. Von der Analyseebene des empirischen Kodierens auf Textebene (Bereich erster Ordnung) wird auf die Strukturiertheit geschlossen, die in der Textebene impliziert ist (Bereich zweiter Ordnung). Diese wird durch die Diskurskodierung erschlossen. Durch die Operation des epistemologischen Bruchs wird vom empirischen Kodieren im Bereich erster Ordnung hin zu den Codes im Bereich zweiter Ordnung abstrahiert (Glasze et al., 2009:293). Von der Ordnung des kodierten Textes wird interpretierend darauf geschlossen, welche Ordnung dieser Binnenlogik zu Grunde liegt.

In einem ersten Analyseschritt das Kodierens werden ebenso wie in der GTM Textpassagen markiert und auf Grundlage eines komparativ-interpretativen Vorgehens entlang von Prinzipien der Ähnlichkeit und Unterschiedlichkeit ein Codebaum aufgebaut (Berg und Milmeister, 2008; Strauss und Corbin, 1998). Diese empirischen Codes bereiten so die Abstraktion der Diskurskodierung vor (Diaz-Bone und Schneider, 2003:474; Parker, 2008:310). Mit fortschreitender Kodierung, also dem Strukturieren und Verbinden von Textpassagen entlang von definierten Kategorien, kann die interpretierende Abstraktion beginnen (Diaz-Bone, 2006:2425).

Marttila (2015a:34 passim, 2015b:134 passim) führt ein heuristisches Kategorienset ein (,phänomenale Identitäten“), das als Richtungsweiser für die Abstraktion von erster auf die Analyseebene zweiter Ordnung dienen kann. Dabei unterscheidet er nach Werten (z.B. ethische Ideale), Subjektivitäten (z.B. Protagonisten, Gegner) und Aktivitäten (z.B. Strategien, Objekte). Es kann sicherlich sinnvoll sein, Marttilas phänomenalen Kategorien zu folgen, bzw. sich davon inspirieren zu lassen - keinesfalls sollten diese aber die Möglichkeiten der Rekonstruktion von diskursiver Strukturiertheit beschränken. Letztlich wird die Strukturiertheit des analysierten Regimes im analytischen Prozess zwischen Ana- lyst*in und Material ko-produziert. Die analytische Rekonstruktionsleistung besteht dabei zum einen darin ein plausibles Kategorienset und damit auch eine plausible Ordnungsstruktur zu abstrahieren. Zum anderen gilt es, plausibel einen Bereich zu definieren, der als hegemonialer Ordnungsbereich für alle Argumentationen des Korpus verstanden werden kann.

Aus der rekonstruktiven Kodieranalyse und der mikroanalytischen Argumentanalyse ergibt sich ein komplementäres Analyseschema mit dem wissenschaftliche Wissensordnungen als Regime der Argumentation herausgearbeitet werden können. Das Analyseschema (Abb. 2) fasst dies nochmals zusammen. Die beiden Analysebewegungen sind dabei ausschließlich analytisch von einander zu trennen. In der empirischen Praxis sind sie komplementär. Es wird zwischen beiden Analysebewegungen kontinuierlich gewechselt. Dadurch lässt sich einerseits treffender kodieren, andererseits ergeben sich die Begründungshorizonte durch die Strukturiertheit der Argumentationen zu einander.

\subsection{Rekontextualisierung}

In einem letzten Schritt der Analyse wird nach den gesellschaftlichen Umständen und der sozialen Einbettung der Ergebnisse der durchgeführten Analyse gefragt. Hintergrund dafür ist, dass auf Grundlage des hier vorgestellten diskursund argumentationstheoretischen Vorgehens keine Aussagen über den Entstehungs- und Wirkungskontext der analysierten Wissensordnung gemacht werden können. Welche Effekte diese auf Konstellationen beteiligter Akteure hat und wie sie normativ privilegierend und benachteiligend wirkt, kann mit dieser Analyse nicht erschlossen werden. Dies wird erst durch die Rekontextualisierung und Diskussion der Produktionsbedingungen der herausgearbeiteten Wissensordnung möglich. So können Machteffekte in den Blick genommen werden (Diaz-Bone, 2006:26; Landwehr, 2009:105 passim) und es lassen sich so normative Rückschlüsse darauf ziehen, warum gerade dieses Wissen auf diese Weise produziert wurde und welchen Interessen es zu Gute kommt - beispielsweise ob die hegemoniale Wissensordnung bestimmte Politiken zu legitimieren vermag und welche Konsequenzen dies hat. Bezüglich der sozialen Rolle von wissenschaftlicher Expertise heißt dies, dass diese erst und gerade durch die Rekontextualisierung herausgearbeitet und qualifiziert werden kann. Der gesellschaftliche Kontext ermöglicht es, die Effekte der Wissensordnung zu diskutieren.

\section{Die hegemoniale Wissensordnung in der Ökologie- und Umweltforschung im westlichen China}

Wie in der Einleitung beschrieben wurde, bewegt sich die Ökologie- und Umweltforschung im Westen Chinas in einem gesellschaftlichen Kontext, der durch soziale Spannungen und bürger*innenschaftliche Widerstände gegen die Po- 


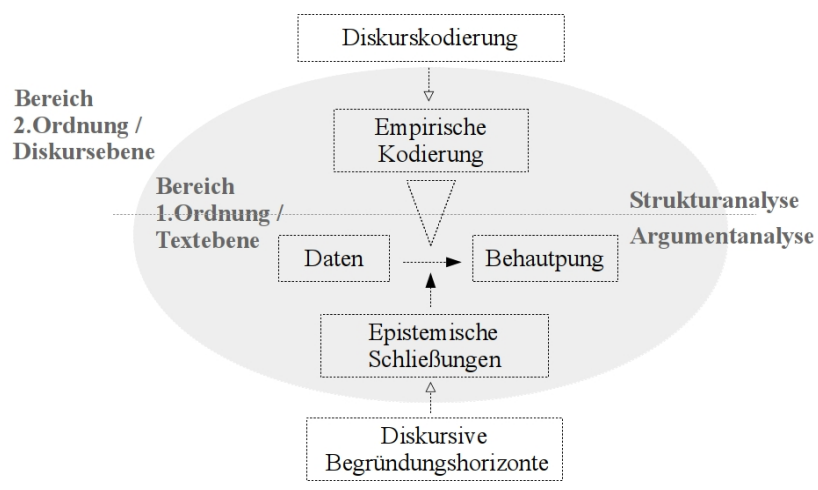

Abb. 2. Argument- und Strukturanalyse, schematisch.

litiken der VRC gekennzeichnet ist. Wie stehen die umweltbezogenen Forschungsarbeiten in dieser Region dazu im Verhältnis und welche Effekte haben diese? Mit dem vorgestellten Vorgehen wurden zur Beantwortung dieser Fragen ca. 100 englischsprachige, wissenschaftliche Kurzbeschreibungen, Zeitschriftenaufsätze und Buchbeiträge aus den Jahren 1999 bis 2013 asynchron untersucht. Dabei wurde deutlich, dass diese Publikationen eine hegemoniale Wissensordnung um das ethische Ideal der „Harmonie“ performieren. Durch die Rekontextualisierung mit nicht-wissenschaftlichen Publikationen (politischen Leit- und Strategiepapieren, Zeitungsbeiträgen, institutionellen Veröffentlichungen) zeigte sich, wie die analysierten Publikationen als Teil der gesellschaftlichen Verhältnisse im Westen Chinas ko-produziert sind und letztlich die territoriale Integrität Chinas und bestehende Machtverhältnisse stabilisieren.

\subsection{Das ethische Ideal der Harmonie als Legitimierungshorizont}

Wie Ernesto Laclau feststellt, strukturieren sich Wissensordnungen um ethische Ideale, die in ihrer Funktion tendenziell bedeutungsleer werden, da sie eine Vielzahl auch mit einander konkurrierender Argumentketten verbinden (vgl. auch Howarth und Stavrakakis, 2000:8; Laclau, 1996:36). Ein solcher tendenziell leerer Signifikant konnte in der durchgeführten Analyse im ethischen Ideal der „Harmonie“ ausgemacht werden. „Harmonie“ steckt den hegemonialen Horizont ab, vor dem sich die Wissensordnung und das wissenschaftliche Arbeiten zu Themen der Ökologie und Umwelt im Westen Chinas entwickelt. In der hier folgenden Darstellung wird nun von dieser höchsten Ordnungsebene der Diskurskodierung (die Ebene, auf die sich die meisten Argumentationsketten beziehen) ausgegangen und dann gezeigt, wie sich dieses Ideal in Argumentationsketten des Regimes weiter ausdifferenziert.

Im Wesentlichen soll durch wissenschaftliches Arbeiten in China dazu beigetragen werden ,eine harmonische Koexistenz und geteilten Wohlstand zwischen Menschen, Natur und Gesellschaft herzustellen“ (Lin und Li, 2011:58; eigene
Übersetzung). Es geht folglich bei wissenschaftlichen Tätigkeiten generell, sowie auch für persönliche Lebensweisen, die sich über ein Ideal der Harmonie gründen darum, das Zusammenleben und die Entwicklung der Welt zu harmonisieren. „Harmonie“ definiert damit sowohl die Teleologie auf die ein gutes, richtiges und gelingendes Leben zu richten ist, als auch die Legitimität und Aufgabe von Wissenschaft, die auf die Harmonie der Welt hinarbeiten soll. Im analysierten Regime äußert sich diese in erster Linie als Ideal sozioökologischer Harmonie. Ökologieforschung trägt dabei zur Harmonisierung des Verhältnisses von „Mensch“ und „Natur" bei.

Folgendes Zitat verdeutlicht die grundlegende Verankerung der Wissensordnung im ethischen Ideal der Harmonie. Ru-zhen Xu schreibt in einer Kurzbeschreibung: „Between humanity and nature, there is a balance, namely harmony. When the balance is broken, the survival of humans will be threatened. Therefore, it is fundamental to adhere to sustainable development to achieve harmony between man and nature" (Xu, 2009, o.S.). Es wird hier argumentiert, das "gute“ und ,richtige“ Verhältnis von „Mensch“ und „Natur“ sei das der „Harmonie“. Umgekehrt sei das Überleben der Menschen, ja der ganzen Menschheit, gefährdet, wenn dieses Verhältnis aus dem Gleichgewicht gerate. Dieses wird hier explizit universalisiert (,Menschheit“). Grundlegend basiert dieses Argument auf der Vorstellung der Trennbarkeit von „Mensch“ und „Natur“. Aus dieser Trennung leitet sich auch die Möglichkeit ab, dieses Verhältnis zu beeinflussen, und es letztlich durch menschliche Aktivitäten ausgestalten zu können. Die implizierte Trennung bereitet den Boden für Argumente der Kontrollierbarkeit dieses Verhältnisses. Damit deutet sich auch an, dass es sich hier nicht um eine „pures“ konfuzianisches Harmonieideal handeln kann. Denn dieses geht von einer harmonisch-verschränkten Einheit von „Mensch“ und „Natur“ aus, aus der sich kein Kontrollanspruch ableiten lässt (Li, 2006). Der Anspruch von Kontrolle, der sich durch die Differenzierung von „Mensch“ und „Natur“ hier eröffnet, kann vielmehr einem europäischaufklärerischen, bzw. modernistischen Begründungshorizont zugeordnet werden. Damit einher geht ein positivistisches Wissenschaftsbild.

Abbildung 3 zeigt, wie sich im Zuge des oben beschriebenen Vorgehens der betreffenden Textstelle genähert werden kann. Freilich lässt sich der Abstraktionssprung zu den Begründungshorizonten aus dem isolierten Zitat alleine nicht eindeutig begründen. Erst im Kontext des gesamten Textkorpus wird diese Abstraktion nachvollziehbar. Ebenso ergibt sich die Diskurskodierung von „Harmonie“ als „ethischem Ideal" aus der Strukturiertheit des Gesamtkorpus. Dort wird „Harmonisierung“ als Verhältnis von Analyse und Kontrollierbarkeit weiter ausdifferenziert und qualifiziert.

Thematisieren wir also weiter diesen Zusammenhang, so wird deutlich, dass die Trennung von „Natur“ und „Mensch“, wie im aufgeführten Zitat skizziert, tatsächlich als Grundlage der Argumentation für Kontrollformen herangezogen wird. 


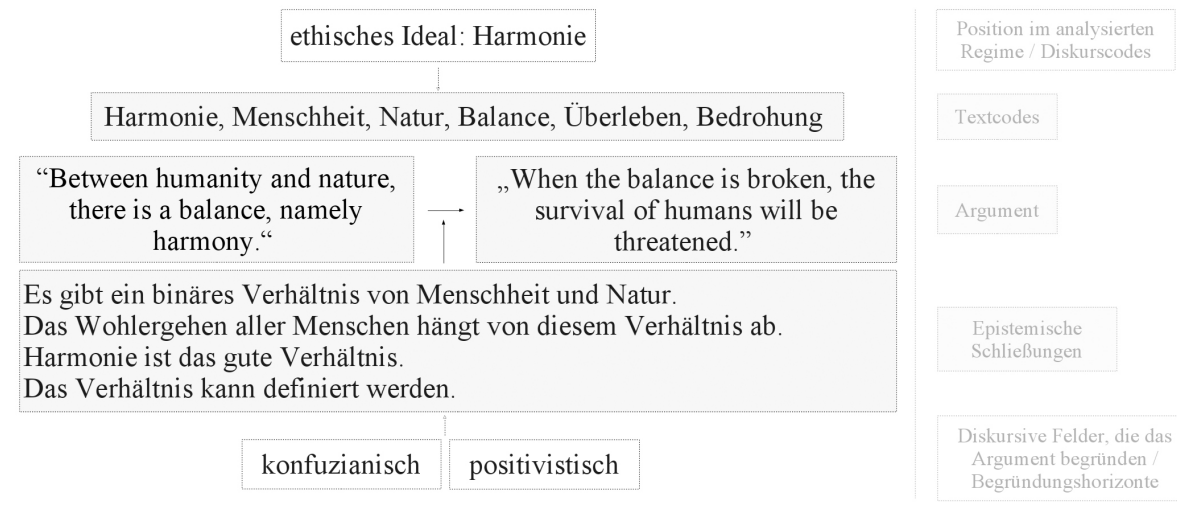

Abb. 3. „Harmonie“; Beispiel des Analyseprozess nach eingeführtem Schema (Abb. 2).

Zhouying (2002:47) formuliert dies für Wissenschaftspraxis in China in dem internationalen Springer Journal „AI \& Society“ so: „Harmonious coexistence and balance are the basic principles for us [...]. Based on this understanding, harmonious coexistence and balance between human behaviour and nature should be implemented“. Die Wortwahl ,implemented" wird von ihm, im vorangegangen Teil des Beitrags spezifiziert [S. 37]: „The essential power of the continuous technology-economy-society system evolution is the ability of human beings or more precisely human wisdom. What makes the system work properly depends on how to coordinate and control human behaviour from macro to micro levels". Sehr deutlich wird hier aus dem Bestreben nach weltlicher Harmonie ein Koordinations- und Kontrollanspruch abgeleitet. Die Verankerung von „Harmonie“ als „basic principle" von Gesellschaft (und damit auch Wissenschaft) wird im Korpus an anderer Stelle gar mit „Harmonieindikatoren“ (z.B. Hou et al., 2011) umgesetzt. Dies zeigt die Aneignung und Ausgestaltung der konfuzianischen Eschatologie der „Harmonie“ als modernes gesellschaftliches und wissenschaftliches Objekt und letzte Sinnstiftungsinstanz von Wissenschaft.

Zwar wird auf den zitierten Beitrag von Zhouying in naturwissenschaftlichen Publikationen nicht direkt Bezug genommen, doch müssen sich die Publikationen der analysierten Umweltforschung vor diesem hegemonialen Horizont positionieren. Textpassagen aus dem Forschungsfeld der Umweltforschung erscheinen damit in einem anderen Licht. Beispielsweise schreiben Xu et al. (2008:60) in der internationalen Elsevier Zeitschrift „Agricultural Water Management“: „In order to promote socially sustainable development, the [water transfer] project [in the Tarim Basin] aims to achieve a balance between humans and the environment as a guideline, sustainable water resource utilization as a core, and ecoenvironmental protection as a base for restoring the degraded ecosystem“. Hier wird ein Forschungsprogramm der sozialökologischen Forschung im Westen Chinas abgesteckt, das sich auf diesen Harmoniehorizont (,sustainable balance“) bezieht, „Defizite“ („,degraded“) identifiziert und für Kon- trolle durch „Interventionen“ (,utilization“, ,protection“) argumentiert. Dieses Schema kehrt auf unterschiedliche Art und Weise im Korpus wieder. Die hegemoniale Wissensordnung wird so weiter eingegrenzt, ausgestaltet und entlang von zwei Argumentationsachsen qualifiziert. Zum einen werden in den Forschungsarbeiten „Defizite an Harmonie“ identifiziert, zum anderen leiten sich daraus spezielle Maßnahmen („Interventionen“) zur Behebung dieser Defizite ab.

Zusammen repräsentieren die Argumentationsketten entlang der Identifikation von „Defizit“ und darauf folgenden spezifischen „Interventionen“ einen Prozess der sozialökologischen Harmonisierung, der gleichzeitig die Forschungsarbeiten selbst legitimiert. Die durch sie erzeugte Wissensordnung weist dabei über den primären Themenbereich der Ökologie hinaus und scheidet Argumentationen entlang der Achsen der Harmonisierung in ,gut“, d.h. harmonisierend, und ,schlecht“" disharmonisierend. Somit werden sowohl bestimmte Repräsentationen von Subjektivitäten, Praktiken und räumlichen Vorstellungen als normative und erstrebenswerte Wirklichkeitsvorstellungen erzeugt und reproduziert, als auch der Argumentraum deutlich, in dem sich Wissenschaftspraktiken bewegen müssen, um als gute Wissenschaft oder vielmehr, als richtige Wissenschaft anerkannt zu sein.

\subsection{Epistemische Eingrenzungen und die Repräsentation von Subjektivität und Räumlichkeit}

Es soll nun deutlicher gemacht werden, wie durch epistemische Eingrenzung Repräsentationen in der hegemonialen Ordnung entlang der Trennung von harmonisierend und disharmonisierend erzeugt werden. Wie bereits angedeutet, wird im hegemonialen Bereich der Wissensordnung ausschließlich über quantitative Indikatoren argumentiert (z.B. „Harmonieindikator") und werden so soziale mit ökologischen Verhältnissen in Beziehung gesetzt. Auf Grundlage von Indikatoren, die ein sozial-ökologisches Defizit repräsentieren, werden Argumente für Interventionsmaßnahmen gestützt. Die Textabschnitte (Abb. 4) von Yu und Zhao 


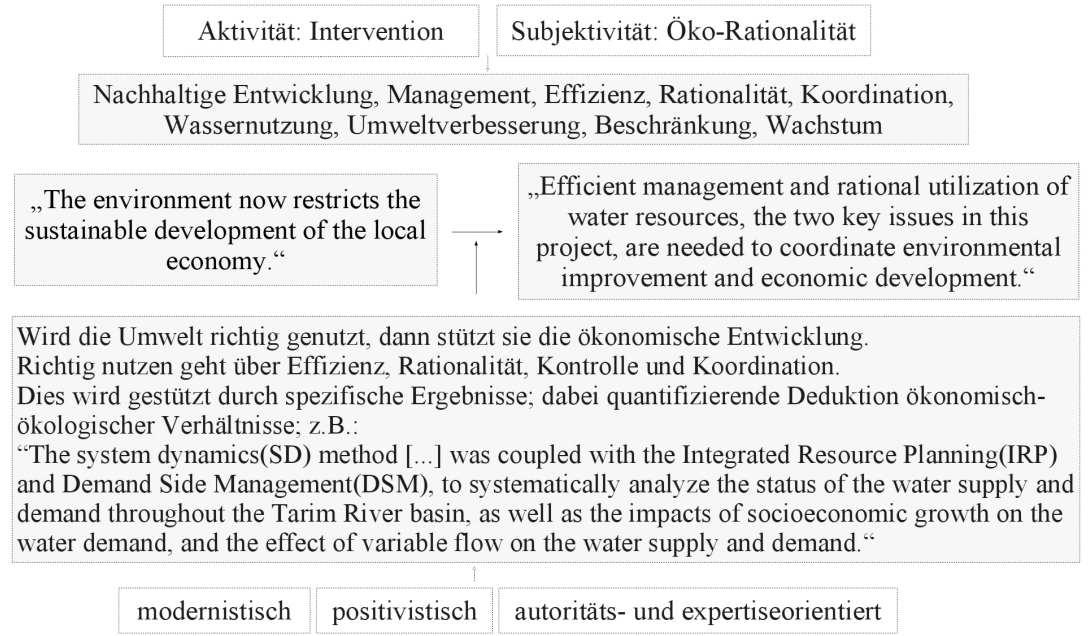

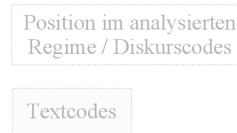

Argument
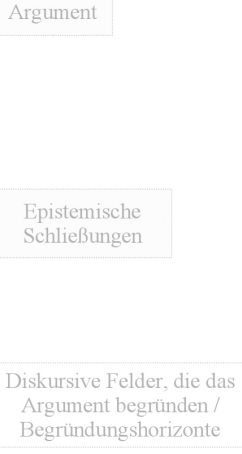

Abb. 4. „Intervention und Rationalität“; Beispiel des Analyseprozess nach eingeführtem Schema (Abb. 2).

(2001:41) zum wiederkehrenden Thema des Wassermanagements verdeutlichen dies: ,The system dynamics (SD) method which can analyze both quantitative and qualitative effects was coupled with the Integrated Resource Planning (IRP) and Demand Side Management (DSM), to systematically analyze the status of the water supply and demand throughout the Tarim River basin, as well as the impacts of socioeconomic growth on the water demand, and the effect of variable flow on the water supply and demand“. Die aufgeführten Indikatoren führen das zuvor ausgeführte Argument des Defizits ,the environment restricts [...]“[S. 39] aus und leiten daraus eine Schlussfolgerung ab: „Efficient management and rational utilization of water resources [...] are needed to coordinate environmental improvement and economic development“ [S. 39]. Es leitet sich mit diesem auf ,objektive“ Indikatoren gestützten Argument die Legitimierung von Maßnahmen ab, die diesem unausgeglichenen Zustand des Mensch-Umwelt-Systems Abhilfe schaffen sollen. Die interventionistischen Maßnahmen zur Behebung werden konkret benannt (,rational utilization“, ,efficient management“, „coordination“).

Die Art und Weise der ,objektiven“ Identifikation des „Defizits“ auf Basis von quantitativen Indikatoren stellt dabei eine voraussetzungsvolle epistemische Schließung dar. Denn das „Faktische“ dieser Indikatoren ist bereits eine nicht-faktisch gegebene Produktion von Daten entlang bestimmter Auswahlkriterien und Vorstellungen. Darauf aufbauend werden dann argumentativ Schlussfolgerungen abgeleitet - eine weitere voraussetzungsvolle Schließung. Prinzipiell aber gäbe es eine Vielfalt an möglichen Schlussfolgerungen die auf die Identifikation defizitärer Mensch-UmweltBeziehungen folgen könnten, z.B. weniger intensiv zu nutzen oder subsistent zu wirtschaften. Neben dem positivistischen Wissenschaftsverständnis und dem modernistischen Diskurs als Grundlage für die ,faktische“ und methodische SchlieBung, kommt in diesem Beispiel ein weiteres Diskursfeld hinzu, das Teile des Arguments logisch werden lässt. Der empirische Code „Koordination“ verweist auf eine Autoritätszentrierung des hegemonialen Regimes. So wird die Harmonisierung der Mensch-Umwelt-Beziehungen als Kontrollaufgabe ,von oben“ repräsentiert, und damit zur Aufgabe von wissenschaftlichen und staatlichen Institutionen. Auch in anderen Publikationen äußert sich diese Autoritätszentrierung, z.B. bei Ling et al. (2012:280): ,It is important for local administrative authorities to produce a better early-warning mechanism against drought and take corresponding measures in an aim to achieve a reasonable allocation of water resources."

Die Identifizierung eines hegemonialen Kernbereichs des Korpus impliziert, dass auch nicht-hegemoniale Argumentbereiche identifiziert werden können. Diese ,alternativen“ Argumentationen bleiben jedoch auf den hegemonialen Bereich bezogen. Die Unterschiede zum hegemonialen Bereich sind dabei, beginnend bei expliziten Gegenargumenten, graduell. Beispielsweise heißt es bei Wei et al. (2010:2156): „The most conservative option would be to discontinue cropping of former grasslands and return the land to lowintensity grazing of pastures. [...] discontinuing cropping would be unacceptable without government intervention“. Die Autoritätszentrierung auf staatliche Eingriffe bleibt hier als Schlussfolgerung wie auch im hegemonialen Bereich des Korpus bestehen. Im Unterschied zu diesem wird hier aber gleichzeitig für eine extensive Nutzung als Harmonisierungsmaßnahme plädiert. Diese Teilabweichungen vom hegemonialen Diskurs machen deutlich, dass letztlich auch andere Schlüsse gezogen und andere „Daten“ erhoben werden können. Sie bekräftigen gleichfalls die hegemoniale Stellung von 
bestimmten Argumentationsketten und machen es möglich einen hegemonialen Bereich zu rekonstruieren.

Dieser hegemoniale Ordnungsbereich ist dabei in hohem Maße epistemisch eingegrenzt. Das heißt, der Bereich, der als (relevante) Forschung gilt und der Horizont, auf den sich das Sagbare der Umweltforschung beziehen muss, ist sehr eng gesteckt. Dies führt dazu, dass entgegen des eigenen wissenschaftlichen Objektivitätsanspruchs, der normative Gehalt dieser Publikationen umso deutlicher hervortritt. Mit „Harmonisierung“ wird ein bestimmter, eingegrenzter Prozess hin zu ,guten und richtigen“ Verhältnissen repräsentiert und erzeugt. Die Publikationen gehen dabei (notwendigerweise) weit über die Abbildung naturwissenschaftlicher Tatsachen hinaus und produzieren bestimmte, normative Vorstellungen von Subjektivität und räumlicher Organisation.

Am Beispiel aus Abb. 4 lässt sich das verdeutlichen. Der zunächst offene Horizont des Ideals „Harmonie“ wird hier auf bestimmte, vermeintlich notwendige Praktiken (,Aktivitäten“) und ihre Träger*innen (,Subjektivitäten“) eingeengt und diese als spezifische Qualitäten erzeugt. Wie sich hier andeutet, solle das gute, ,harmoniefördernde“ Subjekt ökorational handeln. Dies heißt, es solle sein Handeln an Autoritäten der Koordination und des Managements ausrichten. Im Gesamtkontext des Korpus wird deutlich, dass das ,gute" Subjekt traditionelle Praktiken ablehnt und von der Notwendigkeit der Veränderung seiner Praktiken überzeugt ist. Subjekte sollen sich dahin verändern, intensiv mit technischen Lösungen landwirtschaftlich zu arbeiten, externes Autoritätswissen anzuerkennen und umzusetzen und die eigene Tätigkeit als Teil eines großen Ganzen koordinieren zu lassen. Es soll über Märkte agiert und die Konsumgewohnheiten dem entsprechend angepasst werden. Dies zeigt sich beispielsweise auch in diesem Zitat: ,The inefficient and extensive utilization of water resources should be changed towards the rational one in the irrigated regions in the upper reaches. Lining the canals and water-economizing irrigation should be mainly undertaken, the effective utilization ratio of water resources should be increased“ (Fan et al., 2002:107). „Rationalität“ wird hier zum Platzhalter der Harmonisierung von „Natur“ und „Mensch“ und der nationalen Ökonomie.

Eine entscheidende Rolle bei der Legitimierung von Interventionen und der erfolgreichen Produktion dieser Repräsentationen spielen die räumlichen Dimensionen der hegemonialen Wissensordnung. So wird der Westen der VRC vom Osten Chinas abgegrenzt und zu einem „Anderen“ gemacht, um Interventionen zu legitimieren. „In arid areas of western China, LUCC [Land Use Cover Change] of oases is influenced by unique natural and cultural factors and is restricted by resource conditions" (Tang et al., 2007:65). Paradoxerweise wird hier auf Grundlage eines Arguments, das auf geodeterministischen Vorannahmen (,unique“, ,restricted“) beruht, begründet, warum der Westen Chinas besonderer Beobachtung und besonderer Maßnahmen bedarf. „For oases in arid areas, the investigation of LUCC processes and their driving forces is a basis of regional environmental regulati- on and sustainable development" (Tang et al., 2007:66). Der Westen ist auf einzigartige Weise in seiner naturräumlichen Ausstattung beschränkt, die mit gesellschaftlichen Faktoren interagiert. Gerade aber die Einzigartigkeit des Westens, eine Aussage, die auf jeden Ort der Welt zutreffend ist, wird im hegemonialen Regime Teil eines räumlichen „Otherings“ des Westens. Entlang der Analytik der Defizitachse ist das Umweltdefizit insbesondere dem Westen und den für diesen Raum „einzigartigen“ und „typischen“ Subjektivitäten und Praktiken zuzuschreiben. Das zunächst geodeterministische Argument wird hier ebenfalls über das Ideal der Harmonie mit einem modernistischen Diskurs kombiniert. Dabei erweist sich hier dieser Horizont als die harmonische Vereinigung von Ost- und Westchina, die räumliche Repräsentation als die des Nationalstaats. Ähnlich, wie auch bei der Trennung von „Natur“ und „Mensch“, dient die Trennung von „Ost“ und „West“, die dabei fundamental in den Publikationen aufscheint, als Argument dafür eine nationale Einheit (Harmonie eines Mutterlands) (wieder)herstellen zu müssen. Die Standards, die das Verhältnis von Ost- und Westchina harmonisch werden lassen, werden dabei durch den Osten, also in den politischen Zentren der Volksrepublik definiert - denn dort wird in erster Linie nicht ein solches sozialökologisches Defizit verortet. Es zeigt sich so deutlich, wie sich die Ökologieforschung über Räumlichkeitsargumentationen auf einen nationalistischen Diskurs bezieht, der durch das ethische Ideal der Harmonie gebunden und in ein Verhältnis zu den Mensch-Umwelt-Beziehungen im hegemonialen Regime gesetzt wird. Es geht danach darum, spezifische Interventionen im Bereich sozial-ökologischer Verhältnisse im Westen Chinas zu legitimieren.

Diese kurze Diskussion zeigt, wie in der hegemonialen Wissensordnung nicht nur ein normatives Bild des richtigen Umgangs und Verhältnisses von „Menschen“ und „Natur“ erzeugt wird, sondern wie darüber hinaus bestimmte Vorstellungen des „Guten“ (mit)produziert werden. Dabei werden Repräsentationen erzeugt, die Subjekte dazu anhalten, sich selbst zu diesen guten Subjekten im Sinn der Ordnung zu entwickeln, um nicht die sozial-ökologische Harmonie zu gefährden. Diese ist gleichzeitig die Harmonie eines*r jeden und der VRC selbst. Das gute Subjekt und seine Praxis ist öko-rational, marktwirtschaftlich, technikbegeistert, autoritätsergeben und national orientiert.

\section{3 Öko-gouvernmentale Verschiebungen und die Förderung territorialer Kontrolle}

Die Rekonstruktion der diskursiven Strukturiertheit der Argumentationslinien und ihrer Begründungshorizonte zeigt wie in den analysierten Publikationen ein spezifisches und exklusives Weltverstehen objektiviert wird. Um herauszuarbeiten, welche Effekte und welche Hintergründe die hegemoniale Wissensordnung in ihrem Wirkungskontext hat, wird der Blick bei der Rekontextualisierung vom Korpus wissenschaftlicher Publikationen auf deren Produktionsbe- 
dingungen verschoben. Dies ist wichtig, um Effekte auf unterschiedliche (Subjekt-)Positionen in der Struktur der objektivierten Wissensstruktur thematisieren und normativ benennen zu können (Kapitel 3.3). Dazu wurde hier sowohl mit nicht-wissenschaftlichen Publikationen (Zeitungsartikel, politische Leitpapiere, etc.) als Referenzfläche gearbeitet, als auch die institutionellen Affiliationen der Autor*innen, die akademische Institutionslandschaft und Wissenschaftsförderung Chinas diskutiert. Insbesondere die konflikthafte historische und aktuelle gesellschaftspolitische Stellung des Westen Chinas spielen eine entscheidende Rolle bei der Einordnung des Regimes.

Im Kontext der umkämpften gesellschaftlichen Verhältnisse und der Infragestellung des chinesischen Nationalstaats durch tibetische und uighurische Unabhängigkeitsbewegungen im Westen der Republik (siehe Kapitel 1) beziehen die analysierten, hegemonialen Wissensordnungen eindeutig Position. Letztlich stärken sie die Bemühungen der VRC um territoriale Kontrolle. Der antagonistische Konflikt zwischen den Territorialitäts- und Kontrollansprüchen des Staates einerseits und der widerständigen Bevölkerung andererseits wird im Gewand sozial-ökologischer Forschung ökogouvernmental verschoben. Dies geschieht auf zwei Ebenen.

Erstens werden die bestehenden gesellschaftlichen Konflikte in den Bereich der naturwissenschaftlich belegten Alternativlosigkeit und einer daraus abgeleiteten sozialen Notwendigkeit interventionistischer Maßnahmen verlegt. Dies trifft insbesondere auf vom Staat durchgeführte regulative Maßnahmen zu. Der soziale Charakter und die sozialen Effekte von Interventionen werden durch die Wissensordnung $\mathrm{zu}$ objektivierten und nicht-verhandelbaren Interventionen. Staatliches Handeln wird legitimiert und staatliche Verantwortbarkeit an wissenschaftliche Standards abgegeben. Dies verschiebt die Möglichkeit der Adressierung von Unzufriedenheit von administrativen und exekutiven staatlichen Stellen auf wissenschaftliche Institutionen.

Zweitens werden die antagonistischen Konfliktlinien zwischen Staat und Bürger*innen in die Subjekte selbst verschoben. Das gute Subjekt soll danach nicht für den Staat, sondern für sich selbst und die „Natur“ ökologisch integer handeln. Der Bereich dieser Integrität ist dabei aber implizit deckend mit der Integrität des Staates.

Als Schlüsselträger dieser ökogouvernmentalen Territorialitätsstrategie fungieren dabei auch die räumlichen Repräsentationen des Westens vis-à-vis des Ostens Chinas und die Kulturalisierungen, die damit einhergehen. In der spezifisch chinesischen Aneignung und Fortschreibung des eurozentristisch-kolonialen Diskurses, operiert der Osten und seine Attribute (,proper China“, ,rational“, ,effizient“", „entwickelt“, „hochkulturell“, ,zentral“) als stiller, erstrebenswerter Referent für den Westen Chinas (,ineffizient", ,,irrational“, „traditionell“, „zurückgeblieben“, „exotisch“, „peripher"). Die wissenschaftliche Wissensordnung hat damit den Effekt, den Westen Chinas kulturell und materiell am Osten Chinas auszurichten. Insbesondere wird dies durch das Ideal der „Harmonie“ repräsentiert, an dem sich die hegemoniale Wissensordnung orientiert. So werden nicht nur vermeintlich nicht-ökologische Subjektivierungen und ihre Praktiken und Lebensweisen delegitimiert, sondern eine (moderne) han-chinesische Subjekthaftigkeit, die Elemente des europäisch-aufklärerischen, modernistischen Diskurses integriert, fördert und naturalisiert. Damit wird insbesondere eine Schwächung und Delegitimierung nicht hanchinesischer, im Speziellen uighurischer und tibetischer, Subjektivierungsmöglichkeiten gefördert. Ökologische Integrität wird so als eine kulturelle Disposition erzeugt, die deckungsgleich mit den Subjektivierungsweisen moderner, han-chinesischer und nationalstaatlich orientierter Subjekte ist.

Die Herausforderung der territorialen Kontrolle der VRC im Westen Chinas wird so zu einer Angelegenheit subjektiver und wissenschaftlich organisierter ,sozial-ökologischer Harmonie“. Ein solches Ideal sozial-ökologischer Integrität stellt dabei aber kein Gegenüber eines Konflikts dar, gegen sie kann nicht gekämpft oder sich organisiert werden. Letztlich wirken die hegemonialen Repräsentationen dieser wissenschaftlichen Wissensordnung entmächtigend für Subjektpositionen, die sich nicht am Idealbild des modernen, hanchinesischen Subjekts orientieren und entmächtigend für alle Bestrebungen die sich gegen staatliche und andere Interventionen richten. So werden gleichzeitig Subjektpositionen ermächtigt, die sich an diesem Idealbild orientieren. Die analysierte Wissensordnung artikuliert damit insbesondere auch Ideale, die sich in den politischen Programmen (z.B. im 11. Fünf-Jahres-Plan) der VRC wiederfinden. Ökologische Problembereiche und Lösungsansätze werden im Sinne der Formung einer harmonischen, sozialistischen Gesellschaft repräsentiert (vgl. Fan, 2006:709). Die Wissensordnung trägt damit dazu bei die bestehenden Verhältnisse zu (re)produzieren.

Auffällig ist dabei, dass die hegemonial argumentierenden Publikationen fast ausschließlich von Wissenschaftler*innen der CAS oder in Kooperation mit dieser durchgeführt wurden. Ob die Autor*innen dabei strategisch die dominanten Diskurse reproduzieren, kann im Rahmen dieser Analyse nicht bewertet werden. Jedoch konnte durch die Analyse indirekt gezeigt werden, wie durch vermeintlich objektives, wissenschaftliches Wissen die bestehenden Machtverhältnisse reproduziert werden und die Forschungsarbeiten implizit eine Position in einem diskursiven Konfliktfeld beziehen. Konkret werden hier sozial-kulturelle Konflikte in einen sozial-ökologischen Argumentationsraum verschoben und damit letztlich die staatliche Integrität und territoriale Kontrolle gestützt.

\section{Wissenschaft, Relevanz, Kontrolle}

Mit Kodierungstechniken und einer Mikroanalyse aufbauend auf den Argumentationsanalysen von Toulmin, wurden im 
Zuge der hier vorgestellten Analyse ca. 100 wissenschaftliche Kurzbeschreibungen, Zeitschriften- und Buchbeiträge analysiert und deren hegemoniale Wissensordnung herausgearbeitet. In einem weiteren Schritt wurden diese Publikationen der Ökologie- und Umweltforschung im westlichen China rekontextualisiert und auf ihre Produktionsbedingungen und normativen Effekte hin untersucht. Die hier vorgestellte Analyse zeigt ein empirisches Beispiel dessen, was Sergio Sismondo (2009:191) den „scientific state“ nennt. Es zeigt die Verschränkung und Objektivierung der Beziehung von Wissenschaft und Staat in Form einer sozial-ökologischen Wissensordnung. Dabei wird neben den „klassisch“ interventionistischen Paradigmen von „Moderne“, „Markt“ und „staatlicher Autorität“, in dem analysierten Regime um das Ideal der Harmonie zudem ein neoliberales Responsibilisierungsdogma mit spezifisch han-chinesischer Kulturalisierung befördert. Die analysierte Wissensordnung trägt damit dazu bei, die Konflikte des chinesischen Staates mit nichthan-chinesischen und nationalstaatskritischen Bürger*innen im Westen Chinas in diese Subjekte selbst zu verlagern und die umfassende Umstrukturierung der Region mit dem Ziel ökologischer Integrität zu legitimieren. Im Kontext der Herausforderung der staatlichen Institutionen der Volksrepublik China durch diese Bürger*innen im Westen der Republik, wird deutlich, dass die untersuchten Publikationen damit einen Beitrag zur territorialen Kontrolle und kulturellen Umformung des Westens leisten. So wird durch die öko-gouvernmentale Ordnung der wissenschaftlichen Publikationen die staatliche Integrität stabilisiert und eine hanchinesische kulturelle Hegemonie unterstützt.

Die vorliegende Analyse zeigt, wie in einem vermeintlich neutralen und eingrenzbaren Forschungsbereich weit über den Anspruch dieses Bereichs hinaus implizit voraussetzungsvolle und folgenreiche Wissensordnungen (mit)produziert werden. Diese Wissensordnungen sind, wie hier gezeigt werden konnte, nicht zufällig. Vielmehr sind sie Ausdruck der Macht- und Herrschaftsverhältnisse, die ihre Produktion ermöglichen. Über die Ermöglichungsbedingungen wissenschaftlichen Arbeitens sind die gesellschaftlichen Verhältnisse und ihre Vorstellungen in diesem Wissen selbst (mit)angelegt. So zeigt die große Überschneidung von institutioneller Eingebundenheit der Autor*innen einerseits und die Repräsentationen in deren Publikationen andererseits, dass mit der Produktion eines solchen Wissens wohl auch die Bedingungen des eigenen wissenschaftlichen Arbeitens aufrecht erhalten werden. Zwar ist die Wissenschaft in China stärker direkter Kontrolle ausgesetzt, als dies in Nationalstaaten des Globalen Nordens der Fall wäre, doch wirft dieses Fallbeispiel grundsätzlich auch Fragen danach auf, inwiefern wissenschaftliche Expertise in Macht- und Herrschaftsstrukturen involviert ist und welche Diskurse implizit in der Wissensproduktion aufgegriffen werden. Denn Wissenschaft bedarf Förderung, und diese wird dann gewährt, wenn ein Vorhaben als förderungswürdig und anschlussfähig angesehen wird. Die Strukturen der Wissenschaftsförderung sind dabei im Globalen Norden gleichfalls entweder an staatliche Institutionen gebunden oder durch Unternehmen und deren Stiftungen gewährleistet. Über diese Relevanzstrukturen ist wissenschaftliches Arbeiten wie Jasanoff (2005:229) konstatiert, grundlegend einer ,subtilen Kontrolle“ ausgesetzt.

Das vorgestellte argumentationstheoretische Vorgehen bietet dabei eine Möglichkeit diese ,subtile Kontrolle“ über die Analyse und Rekontextualisierung von akademischen Wissensordnungen sichtbarer zu machen. Es lässt sich so mit dem vorgestellten Vorgehen herausarbeiten, welche Wissensordnungen in wissenschaftlichen Argumentationen erzeugt werden, auf welche Weise diese Subjekte in einem Wissensfeld zu einander in Beziehung stehen und in welchem Kontext diese Ordnungen er- bzw. entmächtigend wirken können. Letztlich wird mit dieser Analyse auch deutlich, dass Wissenschaft immer ein ethisch-politisches Unterfangen ist und es nicht möglich ist, im wissenschaftlichen Arbeiten keine Position zu beziehen. Es werden einmal mehr Fragen danach aufgeworfen, warum, wie, und mit welchen Effekten wir Forschungsvorhaben umsetzen und welche wissenschaftliche Expertise dabei produzieren.

Daten-Verfüg barkeit. Der Textkorpus, auf dessen Grundlage der Artikel basiert, ist zu finden unter doi:10.17613/M6ZP8R (Stenglein, 2017).

Folgende Literatur wurde aus dem Textkorpus zitiert: Fan et al. (2002), Lin und Li (2011), Ling et al. (2012), Hou et al. (2011), Tang et al. (2007), Wei et al. (2010), Xu et al. (2008), Xu (2009), Yu und Zhao (2001) und Zhouying (2002).

Interessenkonflikt. Der Autor erklärt, dass kein Interessenkonflikt besteht.

Danksagung. Vielen Dank den Herausgeber*innen Jeannine Wintzer und Anne Vogelpohl für die Geduld und hilfreichen Kommentare im Entstehungsprozess dieses Beitrags und den beiden anonymen Reviewer*innen, die sich zweimal die Zeit genommen haben Kritik zu üben und wichtige Anregungen für die Bearbeitung des Beitrags zu geben.

Edited by: J. Wintzer

Reviewed by: three anonymous referees

\section{Literatur}

Amossy, R.: The New Rhetoric's Inheritance. Argumentation and Discourse Analysis, Argumentation, 23, 313-324, 2009.

Berg, C. und Milmeister, M.: Im Dialog mit den Daten das Erzählen der Geschichte finden, Über das Kodierverfahren der Grounded-Theory-Methodologie, Forum Qual. Sozialforschung, 9, 13, 2008. 
Blaikie, P. M. und Muldavin, J. S. S.: Upstream, Downstream, China, India: The Politics of Environment in the Himalayan Region, Ann. Assoc. Am. Geogr., 94, 520-548, 2004.

Diaz-Bone, R.: Zur Methodologisierung der Foucaultschen Diskursanalyse, Forum Qual. Sozialforschung, 7, 75-85, 2006.

Diaz-Bone, R.: Die französische Epistemologie und ihre Revisionen: Zur Rekonstruktion des methodologischen Standortes der Foucaultschen Diskursanalyse, Hist. Soc. Res., 33, 29-72, 2008.

Diaz-Bone, R. und Schneider, W.: Qualitative Datenanalysesoftware in der sozialwissenschaftlichen Diskursanalyse - Zwei Praxisbeispiele, in: Handbuch Sozialwissenschaftliche Diskursanalyse, Bd. II, Herausgeber: Keller, R., Hirseland, A., Schneider, W., und Viehöver, W., 457-494, Leske + Budrich, Opladen, 2003.

Eemeren, F. H. van, Garssen, B., Krabbe, E. C. W., Henkemans, F. A. S., Verheij, B., und Wagemans, J. H. M.: Handbook of Argumentation Theory, Springer, New York, 2014.

Evagorou, M. und Osborne, J.: The role of language in the learning and teaching of science, in: Good Practice In Science Teaching: What Research Has To Say, Herausgeber: Osborne, J. und Dillon, J., Open University Press, Maidenhead, 2010.

Fan, C. C.: China's Eleventh Five-Year Plan (2006-2010): From "Getting Rich First" to "Common Prosperity", Eurasian Geogr. Econ., 47, 708-723, 2006.

Fan, Z., Xia, X., Shen, Y., Alishir, K., Wang, R., Li, S., und Ma, Y.: Utilization of Water resources, ecological balance and land desertification in the Tarim Basin, Xinjiang, Sci. in China (Series D), 45, 103-108, doi:10.1007/BF02878395, 2002.

Felgenhauer, T.: Geographie als Argument, Eine Untersuchung regionalisierender Begründungspraxis am Beispiel ,Mitteldeutschland“, Steiner, Stuttgart, 2007.

Felgenhauer, T.: Raumbezogenes Argumentieren. Theorie, Analysemethode, Anwendungsbeispiele, in Handbuch Diskurs und Raum - Theorien und Methoden für die Humangeographie sowie die sozial und kulturwissenschaftliche Raumforschung, Herausgeber: Glasze, G. und Mattissek, A., 261-278, Transcript, Bielefeld, 2009.

Felgenhauer, T.: Regionalität als Rationalität. Die argumentative Konstruktion von Regionen, Eur. Reg., 21, 47-59, 2015.

Forsyth, T.: Critical Political Ecology: The politics of environmental science, Routledge, London und New York, 2003.

Forsyth, T. und Walker, A.: Forest Guardians, Forest Destroyers: The Politics of Environmental Knowledge in Northern Thailand, University of Washington Press, Seattle, 2008.

Foucault, M.: Archäologie des Wissens, Suhrkamp, Frankfurt a. M., 1981.

Glasze, G. und Mattissek, A.: Handbuch Diskurs und Raum: Theorien und Methoden für die Humangeographie sowie die sozial- und kulturwissenschaftliche Raumforschung, Herausgeber: Glasze, G. und Mattissek, A., Transcript, Bielefeld, 2009.

Glasze, G. und Mattissek, A.: Diskursforschung in der Humangeographie, in Diskursforschung, Herausgeber: Angermuller, J., Nonhoff, M., Herschinger, E., Macgilchrist, F., Reisigl, M., Wedl, J., Wrana, D., und Ziem, A., transcript Verlag, Bielefeld, 2014.

Glasze, G., Husseini, S., und Mose, J.: Kodierende Verfahren in der Diskursforschung, in: Handbuch Diskurs und Raum - Theorien und Methoden für die Humangeographie sowie die sozial und kulturwissenschaftliche Raumforschung, Herausgeber: Glasze, G. und Mattissek, A., 293-314, Transcript, Bielefeld, 2009.
Hou, Y., Maimaitiming, A., und Hou, Z.: Analysis on harmony degree of water resources utilization based on fuzzy theory: a case study of Bortala Mongol Autonomous Prefecture, Water Res. Protect., 3, abstract, verfügbar unter: http://en.cnki.com. cn/Article_en/CJFDTotal-SZYB201103000.htm (aufgerufen am 05. Dezember 2012), 2011.

Howarth, D. und Stavrakakis, Y.: Introducing Discourse Theory, in: Discourse Theory and Political Analysis: Identities, Hegemonies and Social Change, Herausgeber: Howarth, D., Stavrakakis, Y., und Norval, A., Manchester University Press, Manchester, 2000.

Jasanoff, S.: Judgement under Siege: The Three-Body Problem of Expert Legitimacy, in: Democratization of Expertise? Exploring Novel Forms of Scientific Advice in Political Decision-Making, Herausgeber: Maasen, S. und Weingart, P., 209-224, Springer, Dordrecht, 2005.

Keith, W. und Rehg, W.: Argumentation in Science: The CrossFertilization of Argumentation Theory and Science Studies, in: Handbook of Science and Technology Studies, Herausgeber: Hackett, E. J., Amsterdamska, O., Lynch, M., und Wajcman, J., 211-240, MIT University Press, Cambridge, 2008.

Laclau, E.: Emancipation(s), Verso, London, 1996.

Laclau, E. und Mouffe, C.: Hegemony and Socialist Strategy, 2. Ed., Verso, London, 2001.

Landwehr, A.: Historische Diskursanalyse, 2. Ed., Herausgeber: Bösch, F., Angelika, E., Gestrich, A., Marszolek, I., Potthast, B., Rau, S., Röckelein, H., Schwerhoff, G., und Wagner-Hasel, B., Campus Verlag, Frankfurt a. M., 2009.

Li, C.: The Confucian Ideal of Harmony, Philos. East West, 56, 583603, 2006.

Lin, X. und Li, X.: Green Transformation in China: From Sustainable Development to Ecological Modernisation. M \& D Forum, 12, 57-60, verfügbar unter: http://www.seiofbluemountain. com/upload/product/201112/2011jscx0a11.pdf (aufgerufen am 02. Dezember 2012), 2011.

Ling, H., Xu, H., Fu, J., und Liu, X.: Surface runoff processes and sustainable utilization of water resources in Manas River Basin, Xinjiang, China, J. Arid. Land, 4, 271-280, doi:10.3724/SP.J.1227.2012.00271, 2012.

Marttila, T.: Post-Foundational Discourse Analysis: A Suggestion for a Research Program, Forum Qual. Sozialforschung, 16, 2015a.

Marttila, T.: Post-Foundational Discourse Analysis: From Political Difference to Empirical Research, Palgrave MacMillan, New York, 2015b.

Mol, A.: Environment and Modernity in Transitional China: Frontiers of Ecological Modernization, Dev. Change, 37, 29-56, 2006.

Parker, I.: Discourse Analysis, in Companion to qualitative Research, Herausgeber: Flick, U., von Kardorff, E., und Steinke, I., 308-312, Sage Publications, London, 2008.

Sismondo, S.: An Introduction to Science and Technology Studies, 2. Ed., John Wiley and Sons Ltd, Chichester, 2009.

Stenglein, F.: Academic Publications on Ecology and Environment in Western China - Qualitative Discourse Analysis Data Set, CORE Repository, doi:10.17613/M6ZP8R, 2017.

Strauss, A. und Corbin, J.: Basics of Qualitative Research: Techniques and Procedures for Developing Grounded Theory, 2. Ed., Sage Publications, London, 1998. 
SuMaRiO: Sustainable Management of River Oases along the Tarim River, verfügbar unter: http://www.sumario.de/de/ (aufgerufen am 3. April 2016), 2016.

Tang, F., Chen, X., Luo, G., Lin, Q., und Liu, H.: A contrast of two typical LUCC processes and their driving forces in oasis of arid areas: A case study of Sangong River Watershed at the nothern foot of Tianshan Mountains, Sci. in China (Series D), 50, Supp.I, 65-75, doi:10.1007/s11430-007-5003-8, 2007.

Tans, O.: The Fluidity of Warrants: Using the Toulmin Model to Analyse practical Discourse, in Arguing on the Toulmin Model, vol. 10, Herausgeber: Hitchcock, D. und Verheij, B., 219-230, Springer, Dordrecht, 2006.

Toulmin, S.: The uses of Argument, überarbeitete Ed., Cambridge University Press, Cambridge, 2003.

Veeck, G., Pannell, C., Smith, C., und Huang, Y.: China's Path and Progress, in: China's Geography. The Dynamics of Political, Economic and Social Change, Herausgeber: Veeck, G., Pannell, C., Smith, C., und Huang, Y., 1-14, Rowman \& Littlefield Publishers, Oxford, 2007.

Wei, Y., White, R., Hu, K., und Willett, I.: Valuing Environmental Externalities of oasis farming in left Banner, Alxa, China, Ecol. Econ., 69, 2151-2157, doi:10.1016/j.ecolecon.2010.05.019, 2010.

Wintzer, J.: Geographien erzählen. Wissenschaftliche Narrationen von Geschlecht und Raum, Franz Steiner Verlag, Stuttgart, 2014.

Wong, E.: Riots in Western China. Amid Ethnic Tension, N. Y. Times, 5. Juli, verfügbar unter: http://www.nytimes.com/2009/ 07/06/world/asia/06china.html?ref=global-home\&_=0 (aufgerufen am 11. August 2016), 2009.
Xu, H., Ye, M., und Li, J.: The water transfer effects on agricultural development in the lower Tarim River, Xinjiang of China, Agric. Water Manag., 95, 59-68, doi:10.1016/j.agwat.2007.09.004, 2008.

$\mathrm{Xu}, \mathrm{R} .:$ On the Harmony between Man and Nature, Jou. of Linyi Normal Univ., 1, abstract, verfügbar unter: http://en.cnki.com.cn/ Article_en/CJFDTOTAL-LQSZ200901023.htm (aufgerufen am 08. Dezember 2012), 2009.

Yeh, E. T.: Green Governmentality and Pastoralism in Western China: "Converting Pastures to Grassland", Nomadic Peoples, 9, 930, 2005.

Yeh, E. T.: Greening western China: A critical view, Geoforum, 40, 884-894, 2009.

Yu, S. und Zhao, X.: Tarim River Water Allocation and CommunityBased Management, in: Irrigation Against Rural Poverty: An Overview of Issues and Pro-Poor Intervention Strategies in Irrigated Agriculture in Asia, Herausgeber: Hussain, I. und Biltonen, E., International Water Management Institute, Colombo, 39-69, verfügbar unter: http://hdl.handle.net/10568/38022 (aufgerufen am 08. Januar 2013), 2001.

Zhou, S.: Building Ecological Civilization and Sustainable Development, Ministry of Envrionmental Protection, PRC, verfügbar unter: http://english.mep.gov.cn/Ministers/Speeches/201208/ t20120807_234449.htm, (aufgerufen am 08. Juni 2016), 2012.

Zhouying, J.: Driving Force for Sustainable Development: Principles of Harmony and Balance, AI \& Soc., 16, 21-48, verfügbar unter: http://link.springer.com/article/10.1007/PL00022693, 2002. 